\title{
EDITORIAL AND COMMENT \\ Personalizing Diabetes Prevention: Is it Time to Focus on the Intervention?
}

\author{
Alka M. Kanaya, MD \\ Division of General Internal Medicine, University of California, San Francisco, San Francisco, CA, USA.
}

J Gen Intern Med 30(11):1570-1

DOI: $10.1007 / \mathrm{s} 11606-015-3409-0$

(c) Society of General Internal Medicine 2015

$\mathrm{T}$ here are approximately 86 million adults with prediabetes in the United States, ${ }^{1}$ and an estimated $70 \%$ of these individuals will develop type 2 diabetes during their lifetime. Several clinical trials have shown a remarkable reduction of diabetes incidence with intensive lifestyle interventions, ${ }^{2}$ but these behavioral changes are difficult to sustain. Less intensive trials testing the real-world effectiveness of behavioral interventions in various settings have found about half the effect on weight loss as the intensive trials. ${ }^{3}$ As we enter this age of personalized medicine, could knowing one's genetic risk motivate patients to make and sustain effective behavioral changes?

In this issue of JGIM, Voils and colleagues tested whether the addition of genetic risk results to standard diabetes risk estimates with brief lifestyle counseling would be more effective than standard diabetes risk estimates with lifestyle counseling alone for weight loss and behavioral outcomes among veterans. ${ }^{4}$ In this well-designed randomized controlled trial conducted in a primary care setting, investigators analyzed three single nucleotide polymorphisms (SNPs) associated with diabetes risk to calculate the genetic risk for the intervention group and categorized their genetic risk as low, moderate, or high. They found that weight, insulin resistance, physical activity and perceived lifetime risk of diabetes did not differ between groups at 3 or 6 months. Daily caloric intake and fat intake were lower in the genetic-risk-informed group at 3 months, but there was no difference at 6 months. This result did not vary among those with high, moderate or low genetic risk in the intervention arm.

A prior trial performed in another primary care setting found similarly unimpressive effects of providing genetic risk information from 36 diabetes risk SNPs to participants enrolled in a diabetes prevention program on short-term outcomes of motivation, program attendance or weight loss. ${ }^{5}$ Other trials testing the effect of communication of genetic risk of disease on risk-reducing behaviors, such as smoking cessation or physical activity, have also been mostly negative. ${ }^{6}$

Why was this the case for the present study? One explanation may be that the trial was underpowered to detect clinically significant effects. The sample size for this study was

Published online May 19, 2015 determined based on the weight loss difference observed between groups from an intensive diabetes prevention trial that required 300 people to be randomized per group. It is possible that a more modest weight loss outcome based on a less intensive intervention such as the proposed method would have required a larger sample size. While 601 participants were randomized, only 534 (89\%) attended the risk counseling session and only $84 \%$ completed the primary endpoint evaluation at 3 months, which further diminished the study's power. Yet the real question is whether the knowledge of high (or moderate/high) genetic risk will motivate individuals to adopt healthier behaviors. The power to detect an interaction by genetic risk category was very low in this study (only $24 \%$ had high genetic risk and another $37 \%$ had moderate risk in the intervention arm), and this may have yielded the negative result. However, it may be unethical to randomize only those with high genetic risk for diabetes to be informed about this risk, compared to withholding this information to determine whether this knowledge can influence behavior change.

A second explanation for the negative result is that providing patients with genetic risk predisposition for diabetes does not motivate them to change their behavior. As the authors discuss, this may be because the genetic risk is not that strong and therefore not as impactful as it may have been if it was the leading risk factor for diabetes. In fact, studies that have evaluated genetic risk scores using several genetic markers have found only minimal improvement in the diabetes prediction beyond that of standard risk factors. ${ }^{7}$ If the genetic risk is not that strong, than why should this be a motivating factor for behavior change? Should we be conducting studies of an impotent intervention?

Alternatively, regardless of clinical validity, if an individual believes that their genetic risk is high and that diabetes may be an unavoidable prophecy, they may take the fatalistic route and do nothing to prevent diabetes. Or they may believe that a stronger remedy than behavioral change would be required to counteract this risk. While the counselors in this study were careful to tell the participants that lifestyle modification can prevent or delay diabetes even if they have a high genetic risk, the recommended lifestyle modification may not have been perceived as an adequate intervention. In a study of patients with a known genetic disorder of familial hypercholesterolemia, most reported near perfect adherence with cholesterol-lowering 
medication, but only half followed diet and physical activity recommendations. ${ }^{8}$ Perhaps a more personalized intervention would have yielded better results?

On 20 January 2015, President Obama announced the Precision Medicine Initiative "to bring us closer to curing diseases like cancer and diabetes..." 9 The longer-term focus of this initiative is on chronic diseases such as type 2 diabetes so that knowledge about individual variability is used for assessing disease risk, understanding mechanisms, and determining optimal therapy. Until now, the more common genetic variants explain very little of the heterogeneity of type 2 diabetes and do not add much to clinical risk prediction tools. Some hope that large-scale genomic resequencing efforts currently underway will lead to better understanding of specific disease subtypes that will drive the development of new pharmacotherapies, ${ }^{10}$ akin to recent advances in cancer therapy. However, we know that for diabetes prevention, lifestyle changes are more efficacious than pharmacotherapy. Can we further refine the use of proven prevention methods using richer individual information to guide the use of optimal therapies?

A next step in developing a personalized intervention may be to examine existing data and stored biospecimens from large prevention trials to investigate the heterogeneity of the response to lifestyle interventions. What makes high responders different from low responders? Can epigenetic measures such as DNA methylation or extracellular circulating microRNAs give us signals of individuals who are more likely to respond to lifestyle interventions and those who may need to be started on pharmacotherapy instead? Can a combination of genetic and epigenetic information with lifestyle information from these trials provide a more useful guide for tailoring prevention therapies? In future prevention trials, can knowing an individuals' genomic, epigenetic, proteomic, metabolomic, microbiomic, and phenomic information in aggregate help us to improve our prevention efforts? Can we harness the vast quantity of information to better target the behaviors most likely to prevent diabetes?

It has been over a decade since the first large diabetes prevention efficacy trials were completed, and the incidence of diabetes continues to rise. Is this a failure of our health systems in communicating risk information effectively and/or delivering an adequate behavioral intervention? Communication of risk, with or without genetic information, appears to have little power to change behavior, and it may be time to turn our attention to personalizing the diabetes prevention intervention. This may be the next frontier for diabetes prevention research.

Conflict of Interest: The author has no conflict of interest to report.

Corresponding Author: Alka M. Kanaya, MD; Division of General Internal MedicineUniversity of California, San Francisco, San Francisco, CA, USA (e-mail: Alka.Kanaya@ucsf.edu).

\section{REFERENCES}

1. Centers for Disease Control and Prevention. http://www.cdc.gov/diabetes/prevention/prediabetes.htm. Accessed 3/24/2015.

2. Baker MK, Simpson K, Lloyd B, et al. Behavioral strategies in diabetes prevention programs: a systematic review of randomized controlled trials. Diabetes Res Clin Pract. 2011;91:1-12.

3. Dunkley AJ, Bodicoat DH, Greaves CJ, et al. Diabetes prevention in the real world: effectiveness of pragmatic lifestyle interventions for the prevention of type 2 diabets and of the impact of adherence to guideline recommendations: a systematic review and meta-analysis. Diabetes Care. 2014;37:922-33.

4. Voils CI, Coffman CJ, Grubber JM, et al. Does type 2 diabetes genetic testing and counseling reduce modifiable risk factors? A randomized controlled trial of veterans. J Gen Intern Med. 2015. doi:10.1007/ s11606-015-3315-5.

5. Grant RW, O'Brien KE, Waxler JL, et al. Personalized genetic risk counseling to motivate diabetes prevention. Diabetes Care. 2013;36:13-9.

6. Marteau TM, French DP, Griffin SJ, et al. Effects of communicating DNAbased disease risk estimates on risk-reducing behaviours. Cochrane Database Syst Rev. 2010;10, CD007275. doi:10.1002/14651858. CD007275.pub2.

7. Meigs JB, Shrader P, Sullivan LM, et al. Genotype score in addition to common risk factors for prediction of type 2 diabetes. N Engl J Med. 2008;359(21):2208-19.

8. Claassen L, Henneman L, Kindt I, et al. Perceived risk and representations of cardiovascular disease and preventive behavior in people diagnosed with familial hypercholesterolemia: a cross-sectional questionnaire study. J Health Psychol. 2010;15(1):33-43.

9. Collins FS, Varmus H. A new initiative on precision medicine. N Engl J Med. 2015;372(9):794-5.

10. McCarthy MI. Genomics, type 2 diabetes, and obesity. N Engl J Med. 2010;363(24):2339-50. 